\title{
Leveraging Methylation Alterations to Discover Potential Causal Genes Associated with the Survival Risk of Cervical Cancer in TCGA through a Two- stage Inference Approach
}

Jinhui Zhang

Xuzhou Medical College: Xuzhou Medical University https://orcid.org/0000-0002-7453-2283

\section{Ting Wang}

Xuzhou Medical College: Xuzhou Medical University

\section{Xinghao Yu}

Suzhou University: Soochow University

\section{Shuiping Huang}

Xuzhou Medical College: Xuzhou Medical University

Huashuo Zhao

Xuzhou Medical College: Xuzhou Medical University

Ping Zeng ( $\sim$ zpstat@xzhmu.edu.cn )

School of Public Health, Xuzhou Medical University https://orcid.org/0000-0003-2710-3440

\section{Research article}

Keywords: cervical cancer, The Cancer Genome Atlas, causal gene, two-stage inference, instrumental variable, DNA methylation, gene expression, prediction model, harmonic mean p-value combination method, cox linear mixed-effects model

Posted Date: October 6th, 2020

DOI: https://doi.org/10.21203/rs.3.rs-80103/v1

License: (c) (i) This work is licensed under a Creative Commons Attribution 4.0 International License. Read Full License

Version of Record: A version of this preprint was published at Frontiers in Genetics on June 2nd, 2021. See the published version at https://doi.org/10.3389/fgene.2021.667877. 


\section{Leveraging methylation alterations to discover potential}

2 causal genes associated with the survival risk of cervical

3 cancer in TCGA through a two-stage inference approach

5 Jinhui Zhang ${ }^{{ }^{*}}$, Ting Wang ${ }^{*}$, Xinghao Yu ${ }^{1}$, Shuiping Huang ${ }^{1,2}$, Huashuo Zhao ${ }^{1,}$

$6 \quad 2$, Ping Zeng ${ }^{1,2}$

7

8 1. Department of Epidemiology and Biostatistics, School of Public Health, Xuzhou

9 Medical University, Xuzhou, Jiangsu, 221004, China

10 2. Center for Medical Statistics and Data Analysis, School of Public Health, Xuzhou

11 Medical University, Xuzhou, Jiangsu, 221004, China

13 * The authors wish it to be known that, in their opinion, the first two authors should be 14 regarded as joint first authors.

Correspondence Authors: Huashuo Zhao and Ping Zeng

17 Address: Department of Epidemiology and Biostatistics, School of Public Health,

18 Xuzhou Medical University, Xuzhou, Jiangsu, 221004, China.

19 Tel: +86 13305218786; E-mail: hszhao@xzhmu.edu.cn

20 Tel: +86 15996970535; FAX: +86 15996970535; E-mail: zpstat@ xzhmu.edu.cn 
Background: Multiple genes were previously identified to be associated with cervical cancer; however, the genetic architecture of cervical cancer remains unknown and many causal genes have yet been discovered.

Methods: To explore causal genes related to cervical cancer, a two-stage causal inference approach was proposed within the framework of Mendelian randomization, where the gene expression was treated as exposure, with methylations located within that gene serving as instrumental variables. Five prediction models were first utilized to characterize the relationship between the expression and methylations for each gene; then the methylation-regulated gene expression (MReX) was obtained and the association was evaluated via Cox mixed-effects model based on MReX. We further implemented the harmonic mean $p$-value (HMP) combination to take advantage of respective strengths of these prediction models while accounting for dependency among the $p$-values.

Results: A total of 14 causal genes were discovered to be associated with the survival risk of cervical cancer in TCGA when the five prediction models were separately employed. The total number of causal genes was brought to 23 when conducting HMP. Some of the newly discovered genes may be novel (e.g. YJEFN3, SPATA5L1, IMMP1L, C5orf55, PPIP5K2, ZNF330, CRYZL1, PPM1A, ESCO2, ZNF605, ZNF225, ZNF266, FICD and OSTC). Functional analyses showed these genes were enriched in tumor-associated pathways. Additionally, four genes (i.e. COL6A1, SYDE1, ESCO2 and GIPC1) were differentially expressed.

Conclusion: Overall, our study discovered promising candidate genes that are causally associated with the survival risk of cervical cancer and thus provided new insights into the genetic etiology of cervical cancer.

Keywords: cervical cancer, The Cancer Genome Atlas, causal gene, two-stage inference, instrumental variable, DNA methylation, gene expression, prediction model, 


\section{Background}

50 Cervical cancer is a sexually transmitted disease, mostly caused by infection with 51 human papillomavirus (HPV) [1]. In terms of cancer statistics in 2018, cervical cancer 52 is the fourth most common malignancy and the fourth leading cause of cancer death 53 among women worldwide, with an estimate of 570,000 cases and 311,000 deaths 54 globally [2]. Moreover, cervical cancer is the second primary cause of cancer death in 55 women aged 20 to 39 years [3]. Although great advanced have been achieved for cervical cancer, reliable diagnostic biomarkers and methods for early identification and screening remain lacking [4, 5]. In addition, despite the unitization of HPV vaccines for prevention and chemoradiotherapy as well as radical surgery offering satisfactory survival rate for early-stage cervical cancer patients, effective treatments for advanced patients are rarely available, especially in developing countries and regions [5].

Therefore, it is an urgent demand in clinical practice that valuable biomarkers should be well discerned and validated to signal the early stage or provide profile of cervical cancer progression [6]. As an effort to understand the genetic foundation of susceptibility to cervical cancer, in the past decade multiple genome-wide association studies (GWASs) were undertaken and discovered a group of cervical-cancer associated genetic variants; see Table S1 for details and see also [5] where a large number of associated germline genetic variants and genes were described for cervical cancer. These findings imply that the development of cervical cancer relies to a significant extend on inherited genetic components and genetic predisposing factors may affect the probability and persistence of, or sensitivity to HPV infection and the rate of tumor development as well as progression [5]. However, like many complex human traits and diseases, the genome-wide SNP-based heritability of cervical cancer estimated in GWAS is smaller than expected. For example, the heritability is $11.7 \%$ $(\mathrm{se}=9.8 \%)$ in a Japanese population [7] and 24.0\% (se =2.9\%) in a Swedish population [8], both of which are lower than that observed in family studies [9]. The remaining missing heritability suggests that a large number of causal genes and genetic variants have yet been discovered and that continuous efforts to identify causative genes for cervical cancer are worthwhile [5].

As well demonstrated in many studies [10-15], mRNA-gene expression measured at 
81 the transcript level influences the progression of complex diseases more directly than other omic measurements. However, the establishment of causal relationship between altered gene expressions and cervical cancer is not straightforward in observation studies due to unknown confounders and possible reverse causation. The latter is of particular concern because we cannot determine whether the regulated gene expressions are the causal factors or the consequence of the development or progression of cervical cancer due to considerably complicated biological network and interaction. Due to this reason, previous studies often aimed to examine association rather than causality between gene expression and cervical cancer.

In statistical genetics a powerful statistical tool to determine causal relationship and estimate causal effect of the exposure on the outcome in observational studies is Mendelian randomization (MR), which is built based on commonly used instrumental variable approaches developed in the field of causal inference [16-18]. Under some certain assumptions, the results of MR analysis are less susceptible to reverse causation and confounding factors [19]. One of key points in MR is to select valid instrumental variables for the exposure (i.e. expression level in our context). Biologically, methylation $\mathrm{GpG}$ sites of a specific gene within the unique function of transcript start site can down-regulate its expression level, and the deregulated expression can further influence the survival of cancer patients [10, 20-22], indicating that methylation alterations play a central role in cancers by regulating expression profile. This motivates us to propose a one-sample two-stage causal inference approach with methylations as instrumental variables of expression to detect causal genes for cervical cancer. This type of two-stage instrumental variable inference is

104 widely employed in many research fields such as sociology, economics [23] and 105 genetic medicine [24]. In addition, the utilization of methylations serving as 106 instruments for causal inference is also commonly seen in recent genomic integrative 107 analyses [25-29].

108 Methodologically, our proposed approach follows the similar principle of prediXcan 109 [30] that was developed recently to identify causal genes for complex diseases with 110 genetic variants serving as instrumental variables in the framework of 111 transcriptome-wide association studies (TWAS) [30-35]. Specifically, in our context 112 we implement a relatively independent two-stage inference procedure (Figure 1): in 
113 the first stage the weights of methylation alterations for each gene are estimated via 114 genetic prediction models; in the second stage the methylation-regulated gene 115 expression $(\mathrm{MReX})$ is imputed with the corresponding predictive model and then the 116 causal association between the gene and the survival risk of cervical cancer is 117 examined based on MReX. More importantly, the two-stage based causal inference 118 can be viewed as a special case of one-sample MR analysis from a statistical 119 perspective [36]. Therefore, under the same conditions of MR our two-stage inference 120 has the ability discovering putatively causal genes for cervical cancer. Furthermore, 121 we consider five commonly used prediction models in the first stage of our two-stage 122 inference procedure and exploit the harmonic mean $p$-values (HMP) method [37] 123 a novel combination strategy that is robust against high correlation $[38,39]-$ to 124 take advantage of respective strengths of these models while accounting for 125 dependency among the $p$-values of various models.

126 We finally apply the proposed approach to the cervical cancer dataset in The Cancer 127 Genome Atlas (TCGA) program [40]. A total of 14 causal genes were discovered to 128 be associated with the survival risk of cervical cancer when the five prediction models 129 were separately implemented. The total number of causal genes was brought to 23 130 when conducting the combination test with HMP. Some of the newly discovered 131 genes were reported in previous literature and differentially expressed between tumor 132 and normal tissues. In addition, functional analyses showed that these genes were 133 enriched in tumor-associated pathways. 
136 Our analysis mainly relied on publicly available datasets of cervical cancer in TCGA 137 [40]. From https://xenabrowser.net/hub/, we obtained clinical features on 317 samples, 138 20,530 RSEM normalized expressions on 308 samples and 485,577 DNA methylation 139 alterations on 312 samples. To avoid racial heterogeneity, we kept 190 white cervical 140 cancer patients with primary solid tumor after filtering out samples with too many 141 missing values. The description of important characteristics of this cervical cancer 142 dataset after filtering is given in Table 1. In our following analysis, we only 143 considered protein-coding genes and determined whether DNA methylation 144 alterations belonged to a given gene according to the TCGA annotation mapping file 145 (i.e. illuminaMethyl450_hg19_GPL16304_TCGAlegacy). Then, each gene 146 expression was quantile-transformed so that it followed a standard normal distribution 147 and each methylation was standardized. Missing values were simply imputed with 148 median. The flowchart for our study is shown in Figure 2.

Linear models predicting gene expression with DNA methylation alterations

150 Let $G$ be an $n$-vector of gene expression levels for the $i^{\text {th }}$ gene measured on $n$ 151 individuals, $\boldsymbol{M}$ be an $n \times p$ matrix for a group of DNA methylations that are located 152 within this gene; note that $p$ varies gene by gene. We apply the following linear model 153 to link $G$ and $\boldsymbol{M}$

$$
G=\boldsymbol{M} \boldsymbol{w}+\boldsymbol{\varepsilon}, \boldsymbol{\varepsilon} \sim N\left(0, \sigma_{\varepsilon}^{2} \mathbf{I}_{n}\right)
$$

155 where $\boldsymbol{w}$ is a $p$-vector for effect sizes of DNA methylations, $\boldsymbol{\varepsilon}$ is an $n$-vector of 156 residual errors following an independent and identical normal distribution with mean 157 zero and variance $\sigma_{\varepsilon}^{2}$ and $\mathbf{I}_{n}$ denotes the $n$-dimensional identical matrix. Because of 158 the possible high-dimensional issue where the number of DNA methylations $p$ is 159 larger than the sample size $n$ (see below), the commonly used least squares method is 160 no longer applicable for estimating $\boldsymbol{w}$. We instead employ several novel models which 161 are specially designed for high-dimensional models, and particularly consider five 162 regressions including linear mixed-effects model (LMM) [41-43], Bayesian sparse 
163 linear mixed-effects model (BSLMM) [44], Latent Dirichlet Process Regression (DPR)

164 [35] as well as Lasso [45] and elastic net (ENET) [46]. Among these methods, LMM,

165 BSLMM and DPR explicitly incorporate all DNA methylations into the model by

166 assuming diverse prior distributions for the effect sizes; while Lasso and ENET only

167 include some most important DNA methylations with the way of regularization based

168 on variable selection. The details of these models are descripted in [36]. We

169 implement LMM and BSLMM with the GEMMA software (version 0.94), DPR with

170 the DPR software [35], Lasso and ENET with the R glmnet package (version 2.0-18)

171 [47]. Using these models, we can obtain the estimate of effect sizes of DNA

172 methylations (denoted by $\hat{\boldsymbol{w}}$ ) as well as the MReX level $\hat{G}=\boldsymbol{M} \hat{\boldsymbol{w}}$ for each gene.

\section{Cox mixed-effects regression discovering methylation-regulated genes}

174 We now investigate the association between the gene and the survival risk of cervical 175 cancer using the Cox model [48]. Besides the direct gene effect based on MReX $\hat{G}$, 176 we also incorporate the impact of DNA methylation alterations into the survival model to explain possible horizontal pleiotropy [49-54]

$$
\frac{h(t \mid \boldsymbol{X}, \hat{G}, \boldsymbol{M})}{h_{0}(t)}=\exp (\boldsymbol{X} \boldsymbol{a}+\hat{G} \times b+\boldsymbol{M c}), \boldsymbol{c} \sim N\left(0, \sigma_{c}^{2}\right)
$$

179 where $t$ is the observed survival time, $h_{0}(t)$ is an arbitrary baseline hazard function, $\boldsymbol{a}$ $180=\left(a_{1}, a_{2}, \ldots, a_{m}\right)$ is an $m$-vector of effect sizes for available covariates $\boldsymbol{X}, \boldsymbol{b}$ is the effect

181 size for the given gene and is of our primary interest, and $c=\left(c_{1}, c_{2}, \ldots, c_{p}\right)$ is a $182 p$-vector of effect sizes for DNA methylations. Because of the same reason of 183 high-dimensional problem mentioned before, we assume $c$ 's are random effects 184 following a normal distribution with mean zero and variance $\sigma_{c}^{2}$, leading to the Cox 185 linear mixed-effects regression model (denoted by coxlmm) [55]. When $c=0$, or 186 equivalently $\sigma_{c}^{2}=0$, coxlmm shown in (2) reduces into the general Cox model where 187 only the influence of the methylation-driven gene exists. We fit coxlmm with the $\mathrm{R}$ 188 coxme (version 2.2-10) package [56] via the Laplace approximation algorithm based 189 on the second order Taylor series expansion [55]. The significance of MReX is 190 examined through the Wald test $\left(H_{0}: b=0\right): Z=\hat{b} / \sqrt{\operatorname{var}(\hat{b})}$, where $\hat{b}$ is the 
191 estimate of the effect size $b$, with $\operatorname{var}(\hat{b})$ the variance of the estimate $\hat{b}$. The $p$ 192 value of the $Z$ statistic can be easily obtained because it asymptotically follows a 193 standard normal distribution.

194

195

196

198

Harmonic mean p-value method combining dependent p-values

Because multiple prediction models are applied, for each gene we thus yield a set of $p$-value $p_{k}(k=1,2, \ldots, K$; with $K$ the number of the prediction models) according to (2). Unfortunately, the simple and commonly used Fisher's method for aggregating mutually independent multiple tests cannot be exploited due to highly positive correlation among individual tests since they are implemented for the same data set with the similar logic $[57,58]$. Instead, we apply HMP to generate a pooled $p$ value across tests with various prediction models

$$
\begin{aligned}
& p_{\mathrm{HMP}}=\int_{1 / T_{\mathrm{HMP}}}^{\infty} f_{x}\left(x \mid \log T+0.874, \frac{\pi}{2}\right) d x \\
& T_{\mathrm{HMP}}=\left(\sum_{k=1}^{K} \omega_{k}\right) /\left(\sum_{k=1}^{K} \omega_{k} / p_{k}\right)
\end{aligned}
$$

where $\omega_{k}$ represents the non-negative weight for each $p_{k}$ with $\sum_{k=1}^{K} \omega_{k}=1$ and $K=5$ in our study and assume that $\omega_{k}$ is independent of $p_{k} ; f_{x}$ denotes the Landau distribution probability density function. It has been theoretically demonstrated that the complicated dependency among $p$-values has little influence on the final pooled $p$-value in HMP [37], especially on exceedingly small $p$-values which are of particular interest in practice. We implement HMP with equal weights using the harmonicmeanp package (version 3.0) in R [59].

Functional analysis and differential expression analysis for newly identified associated genes

Gene Ontology (GO) and Kyoto Encyclopedia of Genes and Genomes (KEGG) pathway analyses were conducted using the R clusterProfiler package (version 3.16.0) [60]. In addition, to further evaluate the expression profiles of these newly discovered genes, we performed differential expression analysis with 190 cervical tumors and 
216 three normal tissues that were also available from TCGA. After normalization with 217 the trimmed mean of M values (TMM) method, differential expressed genes (DEGs) 218 were screened via the exact test based on quantile-adjusted conditional maximum 219 likelihood estimation [61, 62] implemented in the edgeR package (version 3.30.3) [63, 220 64]. Following previous work [65, 66], DEGs were defined if FDR $<0.05$ and $\mid \log _{2}$ $221 \quad \mathrm{FC}(\mathrm{CT} / \mathrm{CK}) \mid \geq 1.0$. 
224 After quality control we reserved 485,577 DNA methylation GpG sites and 3 clinical covariates (i.e. age of onset, clinical stage, and tumor status) up to 190 cervical cancer patients of European ancestry. To avoid numerical instability, we focused on protein-coding genes which had at least ten methylations. We also first performed the LMM analysis $[44,67,68]$ for each protein-coding gene based on its methylations and selected genes with the phenotypic variance explained by methylations larger than $1 \%$

230 (corresponding to a correlation coefficient of 10\%). The remaining 12,623 genes are 231 referred to as methylation-regulated genes and included in our subsequent analyses

232 (Figure 2). The number of methylation GpG sites across genes ranges from 10 to 2331,062 , with the majority of analyzed genes $(92.0 \%=11,607 / 12,623)$ having 234 methylations less than 50.

Identification of causal genes with Cox linear mixed-effects regression

236 We employed the coxlmm [55] with various prediction models to examine the 237 relationship between $\mathrm{MReX}$ and the survival risk of cervical cancer patients while 238 adjusting for the direct effect of methylations and the confounding effect of clinical covariates. First, we observe that these prediction models display various performance across genes (Figure 3A). Specifically, some prediction models have higher prediction accuracy for some genes but behave less satisfactorily for others. For example, in

242 terms of $R^{2}$, BSLMM behaves well for $38.3 \%$ genes $(=4,834 / 12,623)$, while Lasso,

243 ENET, LMM and DPR have higher $R^{2}$ for $26.82 \%(=3,386 / 12,623), 14.79 \%$ (= $2441,867 / 12,623), 10.92 \%(=1,378 / 12,623)$ and $9.17 \%(=1,158 / 12,623)$ genes, 245 respectively. As expected, the resulting $p$-values of these prediction methods in 246 coxlmm are highly correlated (Figure 3B). For example, the Pearson's correlation of 247 the $p$-values (in a scale of - $\log 10$ ) ranges from 0.63 between DPR-coxlmm and 248 Lasso-coxlmm to 0.96 between LMM-coxlmm and BSLMM-coxlmm.

249 Based on the results of coxlmm, a total of 14 unique associated genes (false discovery 250 rate $[\mathrm{FDR}]<0.05)$ are identified (Table 2). Specifically, we detect three associated 251 genes with DPR-coxlmm, ten associated genes via Lasso-coxlmm and eight 
associated genes through ENET-coxlmm, but do not discover any associated genes using LMM-coxlmm or BSLMM-coxlmm (Figure 4). Among these, six genes (i.e. YJEFN3, SPATA5L1, C5orf55, PPIP5K2, ESCO2 and ZNF225) are simultaneously found by Lasso-coxlmm and ENET-coxlmm, while only one gene (i.e. VPS4B) is simultaneously discovered by DPR-coxlmm and ENET-coxlmm (Figure 3C).

Among these associated genes we find PCMI $\left(\mathrm{FDR}_{\mathrm{ENET}}=0.032\right)$, classified to the cell cycle control network, was previously discovered to be associated with the early stage of cervical cancer [69]. SPR $\left(\mathrm{FDR}_{\text {Lasso }}=0.003\right)$ is located within $1 \mathrm{Mb}$ genetic region of previous GWAS-identified gene ALMS1 [7]. In addition, VPS4B $\left(\mathrm{FDR}_{\mathrm{DPR}}=0.024\right.$ and $\left.\mathrm{FDR}_{\mathrm{ENET}}=0.031\right)$ is a subtype of VPS4 which is the component of the ESCRT machinery and plays an essential role in HPV infectious entry and capsid disassembly [70]. The remaining ten genes (i.e. YJEFN3, SPATA5L1, C5orf55, PPM1A, IMMP1L, ZNF330, PPIP5K2, ESCO2, FICD and ZNF225) are not directly reported to be related to the survival risk of cervical cancer in previous literature. However, for these genes we find suggestive indirect evidence that may support their association with cervical cancer. Specifically, for example, YJEFN3 is a member of the human YJEFN domain containing protein family strongly expressing in Leydig cell tumors and in the fibromas and participates in cholesterol processing and steroid hormone metabolism [71]. SPATA5L1 might play a key role in inhibits ATP Hydrolysis and four-way junction helicase activity and further influences DNA replication and pathogenesis [72, 73]. Smac/DIABLO was expressed de novo in certain subset of cervical tumors [74], while mature Smac/DIABLO was produced on the mitochondrial inner membrane via IMMP1L [75]. PPIP5 kinases (e.g. PPIP5K2) mediate PP-IPs binding, activate casein kinase 2 (CK2) and promote the phosphorylation of the TTT complex, which stimulates DNA-PK/ATM to activate p53 on the cancer cellular [76, 77]. There exists evidence that miR-135b leads to cervical cancer cell transformation [78] and down-regulated miR-135b expression could inhibit the proliferation and invasion of tumor cells by up-regulating PPM1A [79].

HMP to combine different $p$-values

281 As mentioned before, because the $p$-values obtained from coxlmm with diverse 282 prediction models are highly dependent (Figure 3B), we effectively apply HMP to 
283 combine the five $p$ values and generate an overall significance for each gene (Figure 2 284 and Table 2). Nine associated genes are additionally discovered (Figure 3C), including 285 CRYZL1, ZNF605, ZNF266, SNAI1, OSTC, FAM73A, COL6A1, GIPC1 and DCTPP1. 286 Among these genes, five (i.e. SNAII, COL6A1, GIPC1, DCTPP1 and FAM73A) were 287 identified in prior work and SYDE1 locates within $1 \mathrm{Mb}$ generic region of GIPC1 288 (Table S1).

289 Specifically, it is shown that SNAII, along with ZEB1, regulated the 290 epithelial-mesenchymal transition and was then involved in the metastasis of cervical 291 cancer [80]. The up-regulated COL6A1 expression in the tissues of cervical cancer 292 was related to poor clinical prognosis and treated as an important biomarker of 293 cervical cancer progression [81]. The down-regulation of GIPC1 in cervical cancer 294 with HPV-18 infection can lead to the resistance to cytostatic transforming growth 295 factor $\beta$ signaling through TGF $\beta R 3$ destabilization [82]. In addition, DCTPP1 was 296 found to be differentially expressed in normal and cancerous tissues and it was 297 significantly accumulated in the nucleus of cervical carcinoma, implying the 298 important role of DCTPPI under malignant pathology [83]. Family with sequence 299 similarity 73, member A $(F A M 73 A)$ is the down-regulated gene of DNA from 300 exfoliated cervical cells in terms of the HPV-16 variant analysis [84, 85]. CRYZL1 301 contains an reduced nicotinamide adenine dinucleotide (phosphate) $(N A D(P) H)$ 302 binding site which is involved in cellular metabolism, while cervical lesions are 303 associated with cellular metabolic abnormalities [86]. It is previously found that the 304 members of the ZNF family interact with nucleic acids, proteins and small molecules 305 and are involved in a variety of crucial molecular processes in cervical tumor cells at 306 replication, transcriptional and translational levels. Thus, ZNF605 and ZNF266 may 307 be potentially targetable [87-89]. OSTC can regulate gamma-secretase [90] while this 308 secretase affects the ability of HPV pseudo-viruses infection both in human HaCat 309 cells and mouse cells [91].

310 In summary, compared with the tests via individual prediction methods, it is 311 demonstrated that HMP greatly improves statistical power by combining dependent 312 tests and thus identifies more prognosis-associated genes for cervical cancer. Totally, 31323 genes are discovered to be related to the survival risk of cervical cancer, among 314 which 14 genes are likely newly novel genes (i.e. YJEFN3, SPATA5L1, IMMP1L, 
$316 \quad$ FICD and OSTC).

\section{Identification of DEGs, GO and KEGG pathway annotation}

318 In terms of the differential expression analysis, four DEGs are detected among the 23

319 new genes identified above (Figure 5A). In particular, COL6A1 and SYDE1 are 320 up-regulated genes, while ESCO2 and GIPC1 are down-regulated genes (Figure 5B).

321 To explore the potential functions of these genes that may be associated with the 322 tumorigenesis and development of cervical cancer, we performed functional 323 enrichment analysis with GO and KEGG using the R package clusterProfiler (version 324 3.16.0) [60]. The top 5 significantly enriched GO terms of three parts and two KEGG 325 pathways identified are shown in Figure 5C.

326 The GO biological process (BP) terms are remarkably enriched in polyol metabolic 327 process, regulation of biosynthetic process and signaling pathway, chondrocyte 328 differentiation. For the GO cellular component (CC) terms, the target genes are 329 concentrated in the midbody, pericentriolar material, and so on. The molecular 330 function (MF) category was focused on NADP binding, platelet-derived growth factor 331 binding (Table S2). The KEGG enrichment analysis indicates that these genes are 332 remarkably enriched in tumor-associated pathways, including protein export $(P=$ $3330.028)$ and folate biosynthesis $(P=0.032)$ (Figure 5C). The combined action of folate 334 biosynthesis and graft-versus-host disease were demonstrated to be significantly 335 associated with cervical cancer in suit: HLA-DPB1 [92]. The up-regulated 336 differentially expressed genes are mostly associated with cartilage morphogenesis 337 (ontology: BP), collagen trimer (ontology: CC) and extracellular matrix structural 338 constituent conferring tensile strength (ontology: MF). The down-regulated 339 differentially expressed genes are mostly associated with organic hydroxy compound 340 biosynthetic process (ontology: BP) and organic hydroxy compound metabolic 341 process (ontology: BP), dendritic shaft (ontology: CC) (Table S2). The functional 342 enrichment results suggest that these newly discovered causal genes may participate 343 in oncogenicity and tumor progression in cervical cancer through regulating relevant 344 biological processes and critical pathways. 
346 Given the severe health threat among women and little knowledge of genetic basis for 347 cervical cancer, persistent work should be done to discover genes that are causally 348 related to cervical cancer [5]. The present study is one of such efforts with the aim to 349 detect newly causal genes for cervical cancer through integrative genomic methods. 350 The two-stage inference analysis pipeline applied in this work can be considered as a 351 gene-centered integration approach by aggregating omics datasets and clinical 352 information. With the growing high-throughput omics datasets in cancer research over 353 recent years [40], it is well recognized that the utilization of only one single level of 354 genomic measurements is insufficient to completely untangle the etiology of cancer 355 prognosis $[13,40]$. Based on the omics datasets of TCGA measured from multiple 356 platforms, we treated the gene expression as the exposure and the survival time as the 357 outcome to explore causal genes of cervical cancer within the framework of two-stage 358 MR study to avoid the reverse causation.

359 For each gene, under the biologically plausible assumption that the methylation $\mathrm{CpG}$ 360 sites in the gene can regulate gene expression [10, 20-22], we employed these 361 methylations as instrumental variables. Note that, DNA methylations can also 362 influence the survival through alternative mechanisms different from the pathway by 363 the regulation of gene expression; such a phenomenon is referred to as horizontal 364 pleiotropy in MR [49-54] and violates the necessary assumptions of instrumental 365 variable causal inference. To guard against such violation, we also included the direct 366 effect of methylations when inferring the causal relationship between the gene and the survival of cervical cancer.

368 One critical step in our two-stage inference is to evaluate the effect relationship 369 between a group of DNA methylation $\mathrm{CpG}$ sites and the expression level for each 370 gene. The power of the subsequent association performed in coxlmm would greatly 371 depend on how well the prediction model utilized can capture the underlying genetic 372 architecture of the transcriptome [30-35], which can differ in the numbers, effect sizes 373 and effect directions of causal methylation alterations in diverse genes. Therefore, a 374 powerful two-stage inference approach should in the first stage choose a prediction 375 model whose prior effect distribution closely matches the true effect distribution so 
that it can approximate well the genetic architecture of the gene [35, 36, 44]. For example, if DNA methylation alterations have effect sizes following a normal distribution, then LMM-coxlmm would be more powerful; on the other hand, if only a very small fraction of DNA methylation alterations may be predictive for the gene expression, then the test with sparse prediction models (e.g. Lasso-coxlmm and ENET-coxlmm) would be superior. Due to unknown true association patterns, there is no uniformly most powerful test. As a result, the two-stage association test may perform well for one gene, but not necessarily for another.

To leverage the advantage of distinct prediction models to improve power, instead selecting an optimal prediction model, in the present study we considered a wide range of prediction models in our two-stage inference procedure. It can be imaged that the resulting $p$ values would be highly correlated because they are generated with the same data set following the similar logic (Figure 2). The correlation structure of these $p$-values also depends on the true architecture of gene expression, which however is rarely known in advance and is likely to vary from one gene to another across the genome. Therefore, it is desirable to construct an omnibus test that integrates the advantage of multiple prediction approaches and is robust against distinct transcriptomic architectures. To achieve this aim, we exploited HMP [37] combining these correlated $p$-values and integrating individual strengths of various tests. As illustrated in our empirical application, HMP achieves relatively higher power since it aggregates genetic association information across different tests.

\section{Compared to previous similar methods, the proposed two-stage inference approach} differs in three aspects. First, unlike prediXcan [30] we constructed the two-stage inference procedure in one sample, leading to the so-called one-sample two-stage regression [24]. Second, multiple competing prediction models rather a single model were utilized and combined with HMP which was $p$-value calibrated [37]. Thus, our strategy often has higher power compared to the test with single prediction model. Third, due to widespread pleiotropic effects in omics [49-54], we also considered the direct influence of methylations. Therefore, our results would be robust against the bias of pleiotropy of instrumental variables that are commonly encountered in MR.

However, the present study is not without limitation. First, the methylation-regulated 
genes were analyzed only in TCGA; no external relevant expression profiles were 408 applied for further validation. Second, we only employed methylations as 409 instrumental variables, other omic measurements that regulate gene expression (e.g. 410 genetic variants $[43,93,94])$ can be also simultaneously incorporated to further 411 improve power. Third, we only utilized local methylation GpG sites of a gene as 412 candidate instruments. It is not known whether the power can be further enhanced if 413 the global methylation GpG sites are exploited. Fourth, the present study assumed a 414 linear relationship for each methylations-expression pair. While a linear relationship 415 can be methodologically interpreted as a first-order approximation to non-linear 416 relationship [44], modeling a linear relationship may be suboptimal and suffer from 417 power loss if the true relationship is non-linear. Fifth, due to the complicated standard 418 error structures for those prediction models, in terms of the assumption of no 419 measurement error (NOME) [51], we did not incorporate the uncertainty in the 420 estimated effect sizes of methylations into our two-stage approach, although such 421 uncertainty may be important in integrative genomic causal inference [95, 96]. 422 Actually, we note that many previous two-stage MR studies or TWAS approaches 423 followed this NOME principle [30, 31, 35, 51].

\section{Conclusion}

425 In summary, using the proposed two-stage causal inference approach within the 426 framework of MR analysis, we discovered a total of 14 causal genes which were 427 associated with the survival risk of cervical cancer patients when separately applying 428 five commonly used prediction models. The number of causal genes was brought to

42923 when employing the combination method of HMP. Some may be newly novel 430 genes (i.e. YJEFN3, SPATA5L1, IMMP1L, C5orf55, PPIP5K2, ZNF330, CRYZL1, 431 PPM1A, ESCO2, ZNF605, ZNF225, ZNF266, FICD and OSTC), and some of those 432 newly discovered genes were reported in previous literature and differentially 433 expressed between tumor and normal tissues. In addition, functional analyses showed 434 that those genes were enriched in tumor-associated pathways. Our findings provide 435 new insights into the genetic etiology of cervical cancer and suggest possibly potential 436 therapeutic targets for cervical cancer in the future. 


\section{Supplementary Material}

438 Supplementary material accompanies this paper at BMC Cancer.

439 Additional file 1: Table S1. Genes reported to be associated with risk of cervical 440 cancer through GWAS. Abbreviation: PMID, PubMed ID; OR, Odd Ratio; CI, 441 Confidence Interval.

442 Additional file 1: Table S2. Functional term enrichment analysis by casual genes. 443 Abbreviation: GOID, gene ontology id; BP, biological function; CC, cellular 444 component; MF, molecular function 


\section{ABBREVIATIONS}

446 BP: biological process; BSLMM: Bayesian sparse linear mixed model; Coxlmm: Cox 447 linear mixed-effects model; CC: cellular component; DEG: differential expressed 448 genes; DPR: Latent Dirichlet Process Regression; EBI: the European Bioinformatics 449 Institute; ENET: elastic net; FDR: false discovery rate; GO: Gene Ontology; GWAS: 450 genome-wide association study; HMP: Harmonic mean p-value method; HPV: human 451 papillomavirus; KGEE: Kyoto Encyclopedia of Genes and Genomes; LMM: Linear 452 mixed model; MF: molecular function; MR: Mendelian randomization; TCGA: The 453 Cancer Genome Atlas; TWAS: transcriptome-wide association study

454 Ethics approval and consent to participate

455 Not applicable.

456 Consent for publication

457 Not applicable.

458 Availability of data and materials

459 The datasets used and/or analyzed during the current study are available from the 460 corresponding author on reasonable request or in https://xenabrowser.net/hub/.

\section{Competing interests}

462 The authors declare that they have no competing interests

\section{Funding}

464 This study was partly supported by Youth Foundation of Humanity and Social Science 465 funded by Ministry of Education of China (18YJC910002), Natural Science 466 Foundation of Jiangsu Province (BK20181472), China Postdoctoral Science 467 Foundation (2018M630607 and 2019T120465), Postdoctoral Science Foundation of 468 Xuzhou Medical University, QingLan Research Project of Jiangsu Province for 469 Outstanding Young Teachers, Six-Talent Peaks Project in Jiangsu Province of China 470 (WSN-087), Social Development Project of Xuzhou City (KC19017), National 471 Natural Science Foundation of China (81402765), Statistical Science Research Project 472 from National Bureau of Statistics of China (2014LY112) and Training Project for 473 Youth Science and Technology Innovation Team at Xuzhou Medical University. 
475

476

477

478

479

480

481

482

P.Z. and S.Z. conceived the idea for the study; P.Z., T.W., X.Y. and J.Z. obtained the data and performed the data analyses; P.Z., S.H., T.W. and J.Z. interpreted the results of the data analyses. P.Z., T.W. and J.Z. wrote the manuscript with the participation of all authors.

\section{Acknowledgements}

The TCGA data was publicly available from https://xenabrowser.net/. The data analyses in the present study were supported by the high-performance computing cluster at Xuzhou Medical University.

\section{Author details}

${ }^{1}$ Department of Epidemiology and Biostatistics, School of Public Health, Xuzhou Medical University, Xuzhou, Jiangsu, 221004, China ${ }^{2}$ Center for Medical Statistics and Data Analysis, School of Public Health, Xuzhou Medical University, Xuzhou, Jiangsu, 221004, China *The authors wish it to be known that, in their opinion, the first two authors should be regarded as joint first authors.

\section{References}

1. Šarenac T, Mikov M: Cervical Cancer, Different Treatments and Importance of Bile Acids as Therapeutic Agents in This Disease. Frontiers in pharmacology 2019, 10:484.

2. Bray F, Ferlay J, Soerjomataram I, Siegel RL, Torre LA, Jemal A: Global cancer statistics 2018: GLOBOCAN estimates of incidence and mortality worldwide for 36 cancers in 185 countries. CA: A Cancer Journal for Clinicians 2018, 68(6):394-424.

3. Siegel RL, Miller KD, Jemal A: Cancer statistics, 2019. CA Cancer J Clin 2019, 69(1):7-34.

4. Nakamura K, Sawada K, Yoshimura A, Kinose Y, Nakatsuka E, Kimura T: Clinical relevance of circulating cell-free microRNAs in ovarian cancer. Mol Cancer 2016, 15(1):48.

5. Chen D, Gyllensten U: Lessons and implications from association studies and post-GWAS analyses of cervical cancer. Trends Genet 2015, 31(1):41-54. 
6. Nahand JS, Taghizadeh-Boroujeni S, Karimzadeh M, Borran S, Pourhanifeh MH, Moghoofei M, Bokharaei-Salim F, Karampoor S, Jafari A, Asemi Z et al: microRNAs: New prognostic, diagnostic, and therapeutic biomarkers in cervical cancer. J Cell Physiol 2019, 234(10):17064-17099.

7. Masuda T, Low S-K, Akiyama M, Hirata M, Ueda Y, Matsuda K, Kimura T, Murakami Y, Kubo M, Kamatani Y et al: GWAS of five gynecologic diseases and cross-trait analysis in Japanese. Eur J Hum Genet 2020, 28(1):95-107.

8. Chen D, Cui T, Ek WE, Liu H, Wang H, Gyllensten U: Analysis of the genetic architecture of susceptibility to cervical cancer indicates that common SNPs explain a large proportion of the heritability. Carcinogenesis 2015, 36(9):992-998.

9. Magnusson PKE, Lichtenstein P, Gyllensten UB: Heritability of cervical tumours. Int J Cancer 2000, 88(5):698-701.

10. Wang W, Baladandayuthapani V, Morris JS, Broom BM, Manyam G, Do K-A: iBAG: integrative Bayesian analysis of high-dimensional multiplatform genomics data. Bioinformatics 2013, 29(2):149-159.

11. Zhao H, Ljungberg B, Grankvist K, Rasmuson T, Tibshirani R, Brooks JD: Gene expression profiling predicts survival in conventional renal cell carcinoma. PLoS medicine 2005, 3(1):e13.

12. Kim Y, Kang YS, Seok J: GAIT: gene expression Analysis for Interval Time. Bioinformatics 2018, 34(13):2305-2307.

13. Zhao Q, Shi X, Xie Y, Huang J, Shia B, Ma S: Combining multidimensional genomic measurements for predicting cancer prognosis: observations from TCGA. Briefings in Bioinformatics 2014, 16(2):291-303.

14. Zhu B, Song N, Shen R, Arora A, Machiela MJ, Song L, Landi MT, Ghosh D, Chatterjee $\mathrm{N}$, Baladandayuthapani $\mathrm{V}$ : Integrating clinical and multiple omics data for prognostic assessment across human cancers. Scientific reports $2017,7(1): 16954$.

15. Yu $X$, Wang $T$, Huang $S$, Zeng $P$ : How can gene expression information improve prognostic prediction in TCGA cancers: an empirical comparison study on regularization and mixed-effect survival models. Frontiers in Genetics (in press) 2020.

16. Angrist JD, Imbens GW, Rubin DB: Identification of Causal Effects Using Instrumental Variables. $J$ Am Stat Assoc 1996, 91(434):444-455.

17. Greenland S: An introduction to instrumental variables for epidemiologists. Int J Epidemiol 2000, 29:722-729.

18. Sheehan NA, Didelez V, Burton PR, Tobin MD: Mendelian randomisation and causal inference in observational epidemiology. PLoS Med 2008, 5(8):e177.

19. Davey Smith G, Ebrahim S: 'Mendelian randomization': can genetic epidemiology contribute to understanding environmental determinants of disease? Int J Epidemiol 2003, 32(1):1-22. 
20. Glinsky GV: Integration of HapMap-Based SNP Pattern Analysis and Gene Expression Profiling Reveals Common SNP Profiles for Cancer Therapy Outcome Predictor Genes*. Cell Cycle 2006, 5(22):2613-2625.

21. Fabiani E, Leone G, Giachelia M, D'Alo F, Greco M, Criscuolo M, Guidi F, Rutella S, Hohaus S, Voso MT: Analysis of genome-wide methylation and gene expression induced by 5-aza-2'-deoxycytidine identifies BCL2L10 as a frequent methylation target in acute myeloid leukemia. Leuk Lymphoma 2010, 51(12):2275-2284.

22. de Tayrac M, Lê S, Aubry M, Mosser J, Husson F: Simultaneous analysis of distinct Omics data sets with integration of biological knowledge: Multiple Factor Analysis approach. BMC Genomics 2009, 10(1):32.

23. Angrist JD, Keueger AB: Does Compulsory School Attendance Affect Schooling and Earnings? The Quarterly Journal of Economics 1991, 106(4):979-1014.

24. Xue H, Pan W, for the Alzheimer's Disease Neuroimaging I: Some statistical consideration in transcriptome-wide association studies. Genet Epidemiol 2020, 44(3):221-232.

25. Qi T, Wu Y, Zeng J, Zhang F, Xue A, Jiang L, Zhu Z, Kemper K, Yengo L, Zheng $\mathrm{Z}$ et al: Identifying gene targets for brain-related traits using transcriptomic and methylomic data from blood. Nat Commun 2018, 9(1):2282-2282.

26. Hannon E, Gorrie-Stone TJ, Smart MC, Burrage J, Hughes A, Bao Y, Kumari M, Schalkwyk LC, Mill J: Leveraging DNA-Methylation Quantitative-Trait Loci to Characterize the Relationship between Methylomic Variation, Gene Expression, and Complex Traits. Am J Hum Genet 2018, 103(5):654-665.

27. Liu L, Zeng P, Yang S, Yuan Z: Leveraging methylation to identify the potential causal genes associated with survival in lung adenocarcinoma and lung squamous cell carcinoma. Oncology Letters 2020, 20(1):193-200.

28. Yu $\mathrm{H}$, Cheng $\mathrm{W}$, Zhang $\mathrm{X}$, Wang $\mathrm{X}$, Yue $\mathrm{W}$ : Integration analysis of methylation quantitative trait loci and GWAS identify three schizophrenia risk variants. Neuropsychopharmacology : official publication of the American College of Neuropsychopharmacology 2020, 45(7):1179-1187.

29. Wu Y, Zeng J, Zhang F, Zhu Z, Qi T, Zheng Z, Lloyd-Jones LR, Marioni RE, Martin NG, Montgomery GW et al: Integrative analysis of omics summary data reveals putative mechanisms underlying complex traits. Nat Commun 2018, 9(1):918.

30. Gamazon ER, Wheeler HE, Shah KP, Mozaffari SV, Aquino-Michaels K, Carroll RJ, Eyler AE, Denny JC, Consortium GT, Nicolae DL et al: A gene-based association method for mapping traits using reference transcriptome data. Nature Genetics 2015, 47(9):1091-1098.

31. Gusev A, Ko A, Shi H, Bhatia G, Chung W, Penninx BWJH, Jansen R, de 
Geus EJC, Boomsma DI, Wright FA et al: Integrative approaches for large-scale transcriptome-wide association studies. Nat Genet 2016, 48(3):245-252.

32. Hu Y, Li M, Lu Q, Weng H, Wang J, Zekavat SM, Yu Z, Li B, Gu J, Muchnik $\mathrm{S}$ et al: A statistical framework for cross-tissue transcriptome-wide association analysis. Nat Genet 2019, 51(3):568-576.

33. Wainberg M, Sinnott-Armstrong N, Mancuso N, Barbeira AN, Knowles DA, Golan D, Ermel R, Ruusalepp A, Quertermous T, Hao K et al: Opportunities and challenges for transcriptome-wide association studies. Nat Genet 2019, 51(4):592-599.

34. Barbeira AN, Pividori $\mathrm{M}$, Zheng J, Wheeler HE, Nicolae DL, Im HK: Integrating predicted transcriptome from multiple tissues improves association detection. PLoS Genet 2019, 15(1):e1007889.

35. Zeng $\mathrm{P}$, Zhou X: Non-parametric genetic prediction of complex traits with latent Dirichlet process regression models. Nature Communications 2017, 8(1):456.

36. Zhu $\mathrm{H}$, Zhou $\mathrm{X}$ : Transcriptome-wide association studies: a view from Mendelian randomization. Quantitative Biology 2020.

37. Wilson D: The harmonic mean p-value for combining dependent tests. Proceedings of the National Academy of Sciences 2019, 116(4):1195-1200.

38. Held L: On the Bayesian interpretation of the harmonic mean p-value. Proceedings of the National Academy of Sciences 2019, 116(13):5855-5856.

39. Wilson DJ: Reply to Held: When is a harmonic mean p-value a Bayes factor? Proceedings of the National Academy of Sciences 2019, 116(13):5857-5858.

40. Hoadley KA, Yau C, Hinoue T, Wolf DM, Lazar AJ, Drill E, Shen R, Taylor AM, Cherniack AD, Thorsson V et al: Cell-of-Origin Patterns Dominate the Molecular Classification of 10,000 Tumors from 33 Types of Cancer. Cell 2018, 173(2):291-304.e296.

41. Yang J, Benyamin B, McEvoy BP, Gordon S, Henders AK, Nyholt DR, Madden PA, Heath AC, Martin NG, Montgomery GW et al: Common SNPs explain a large proportion of the heritability for human height. Nature Genetics 2010, 42(7):565-569.

42. Makowsky R, Pajewski NM, Klimentidis YC, Vazquez AI, Duarte CW, Allison DB, de Los Campos G: Beyond Missing Heritability: Prediction of Complex Traits. Plos Genet 2011, 7(4):e1002051.

43. Zeng $\mathrm{P}$, Zhou $\mathrm{X}$, Huang S: Prediction of gene expression with cis-SNPs using mixed models and regularization methods. BMC Genomics 2017, 18:368.

44. Zhou X, Carbonetto P, Stephens M: Polygenic modeling with bayesian sparse linear mixed models. Plos Genet 2013, 9(2):e1003264. 
45. Tibshirani R: Regression shrinkage and selection via the LASSO. Journal of the Royal Statistical Society: Series B (Statistical Methodology) 1996, 58(1):267-288.

46. Zou H, Hastie T: Regularization and variable selection via the Elastic Net. Journal of the Royal Statistical Society: Series B (Statistical Methodology) 2005, 67(2):301-320.

47. Friedman J, Hastie T, Tibshirani R: Regularization Paths for Generalized Linear Models via Coordinate Descent. Journal of Statistical Software 2010, 33(1):1-22.

48. Cox DR: Regression Models and Life-Tables. Journal of the royal statistical society Series B (Methodological) 1972, 34(2):187-220.

49. Verbanck M, Chen C-Y, Neale B, Do R: Detection of widespread horizontal pleiotropy in causal relationships inferred from Mendelian randomization between complex traits and diseases. Nat Genet 2018, 50(5):693-698.

50. Bowden J, Davey Smith G, Burgess S: Mendelian randomization with invalid instruments: effect estimation and bias detection through Egger regression. Int J Epidemiol 2015, 44(2):512-525.

51. Bowden J, Del Greco M F, Minelli C, Davey Smith G, Sheehan NA, Thompson JR: Assessing the suitability of summary data for two-sample Mendelian randomization analyses using MR-Egger regression: the role of the $\mathbf{I}^{2}$ statistic. Int J Epidemiol 2016, 45(6):1961-1974.

52. Burgess S, Thompson SG: Interpreting findings from Mendelian randomization using the MR-Egger method. Eur J Epidemiol 2017, 32(5):377-389.

53. Slob EA, Groenen PJ, Thurik AR, Rietveld CA: A note on the use of Egger regression in Mendelian randomization studies. Int $J$ Epidemiol 2017:dyx 191.

54. Barfield R, Feng H, Gusev A, Wu L, Zheng W, Pasaniuc B, Kraft P: Transcriptome-wide association studies accounting for colocalization using Egger regression. Genet Epidemiol 2018, 42(5):418-433.

55. Therneau TM, Grambsch PM, Pankratz VS: Penalized survival models and frailty. Journal of computational and graphical statistics 2003, 12(1):156-175.

56. Therneau TM: coxme: Mixed Effects Cox Models. $R$ package version 2.2-14. https://CRAN.R-project.org/package=coxme. 2019.

57. Fisher RA: Statistical Methods for Research Workers, 5th Edn. Biological monographs and manuals. Edinburgh: Oliver and Boyd Ltd; 1934.

58. Rice K: A Decision-Theoretic Formulation of Fisher's Approach to Testing. Am Stat 2010, 64(4):345-349.

59. Wilson D: Harmonic Mean p-Values and Model Averaging by Mean $\begin{array}{lllll}\text { Maximum Likelihood. } & R & \text { package } & \text { version } & 3.0 \text {. }\end{array}$ 
https://CRAN.R-project.org/package=harmonicmeanp. 2019.

673

674

675

676

677

678

679

680

681

682

683

684

685

686

687

688

689

690

691

692

693

694

695

696

697

698

699

700

701

702

703

704

705

706

707

708

709

710

711

712

713

60. Yu G, Wang LG, Han Y, He QY: clusterProfiler: an R package for comparing biological themes among gene clusters. Omics : a journal of integrative biology 2012, 16(5):284-287.

61. Robinson MD, Smyth GK: Small-sample estimation of negative binomial dispersion, with applications to SAGE data. Biostatistics 2008, 9(2):321-332.

62. Li CI, Su PF, Shyr Y: Sample size calculation based on exact test for assessing differential expression analysis in RNA-seq data. $B M C$ bioinformatics 2013, 14:357.

63. Robinson MD, McCarthy DJ, Smyth GK: edgeR: a Bioconductor package for differential expression analysis of digital gene expression data. Bioinformatics 2010, 26(1):139-140.

64. McCarthy DJ, Chen Y, Smyth GK: Differential expression analysis of multifactor RNA-Seq experiments with respect to biological variation. Nucleic Acids Res 2012, 40(10):4288-4297.

65. Chai H, Zhou X, Cui Z, Rao J, Hu Z, Lu Y, Zhao H, Yang Y: Integrating multi-omics data with deep learning for predicting cancer prognosis. bioRxiv 2019:807214.

66. Deng S, Ma J, Zhang L, Chen F, Sang Z, Jia Z, Ma L: De novo transcriptome sequencing and gene expression profiling of Magnolia wufengensis in response to cold stress. BMC Plant Biology 2019, 19(1):321.

67. Visscher PM, Hill WG, Wray NR: Heritability in the genomics era--concepts and misconceptions. Nat Rev Genet 2008, 9(4):255-266.

68. Yang J, Manolio TA, Pasquale LR, Boerwinkle E, Caporaso N, Cunningham JM, de Andrade M, Feenstra B, Feingold E, Hayes MG et al: Genome partitioning of genetic variation for complex traits using common SNPs. Nature Genetics 2011, 43(6):519-525.

69. Güzel C, Govorukhina NI, Wisman GBA, Stingl C, Dekker LJM, Klip HG, Hollema H, Guryev V, Horvatovich PL, van der Zee AGJ et al: Proteomic alterations in early stage cervical cancer. Oncotarget 2018, 9(26):18128-18147.

70. Broniarczyk J, Pim D, Massimi P, Bergant M, Gozdzicka-Jozefiak A, Crump C, Banks L: The VPS4 component of the ESCRT machinery plays an essential role in HPV infectious entry and capsid disassembly. Sci Rep 2017, 7:45159.

71. Rudolph C, Sigruener A, Hartmann A, Orso E, Bals-Pratsch M, Gronwald W, Seifert B, Kalbitzer HR, Verdorfer I, Luetjens CM et al: ApoA-I-binding protein (AI-BP) and its homologues $\mathrm{hYjeF}$ _N2 and $h Y j e F \_N 3$ comprise the YjeF_N domain protein family in humans with a role in spermiogenesis and oogenesis. Hormone and metabolic research $=$ Hormonund Stoffwechselforschung = Hormones et metabolisme 2007, 39(5):322-335. 
72. Tanaka AJ, Cho MT, Millan F, Juusola J, Retterer K, Joshi C, Niyazov D, Garnica A, Gratz E, Deardorff M et al: Mutations in SPATA5 Are Associated with Microcephaly, Intellectual Disability, Seizures, and Hearing Loss. American journal of human genetics 2015, 97(3):457-464.

73. White PW, Faucher AM, Massariol MJ, Welchner E, Rancourt J, Cartier M, Archambault J: Biphenylsulfonacetic acid inhibitors of the human papillomavirus type 6 E1 helicase inhibit ATP hydrolysis by an allosteric mechanism involving tyrosine 486. Antimicrobial agents and chemotherapy 2005, 49(12):4834-4842.

74. Martinez-Ruiz G, Maldonado V, Ceballos-Cancino G, Grajeda JP, Melendez-Zajgla J: Role of Smac/DIABLO in cancer progression. Journal of experimental \& clinical cancer research : CR 2008, 27(1):48.

75. Burri L, Strahm Y, Hawkins CJ, Gentle IE, Puryer MA, Verhagen A, Callus B, Vaux D, Lithgow T: Mature DIABLO/Smac is produced by the IMP protease complex on the mitochondrial inner membrane. Molecular biology of the cell 2005, 16(6):2926-2933.

76. Lee S, Kim MG, Ahn H, Kim S: Inositol Pyrophosphates: Signaling Molecules with Pleiotropic Actions in Mammals. Molecules (Basel, Switzerland) 2020, 25(9).

77. Fridy PC, Otto JC, Dollins DE, York JD: Cloning and characterization of two human VIP1-like inositol hexakisphosphate and diphosphoinositol pentakisphosphate kinases. The Journal of biological chemistry 2007, 282(42):30754-30762.

78. Leung CO, Deng W, Ye TM, Ngan HY, Tsao SW, Cheung AN, Pang RT, Yeung WS: miR-135a leads to cervical cancer cell transformation through regulation of $\beta$-catenin via a SIAH1-dependent ubiquitin proteosomal pathway. Carcinogenesis 2014, 35(9):1931-1940.

79. Gao J, Zhang L, Liu Z, Yao S, Gao S, Wang L: Effect of miR-135b inhibitor on biological characteristics of osteosarcoma cells through up-regulating PPM1A. International journal of clinical and experimental pathology 2019, 12(3):689-699.

80. Chen Z, Li S, Huang K, Zhang Q, Wang J, Li X, Hu T, Wang S, Yang R, Jia Y et al: The nuclear protein expression levels of SNAI1 and ZEB1 are involved in the progression and lymph node metastasis of cervical cancer via the epithelial-mesenchymal transition pathway. Human pathology 2013, 44(10):2097-2105.

81. Hou T, Tong C, Kazobinka G, Zhang W, Huang X, Huang Y, Zhang Y: Expression of COL6A1 predicts prognosis in cervical cancer patients. $\mathrm{Am}$ J Transl Res 2016, 8(6):2838-2844.

82. Katoh M: Functional proteomics, human genetics and cancer biology of GIPC family members. Experimental \& molecular medicine 2013, 45(6):e26.

83. Zhang Y, Ye WY, Wang JQ, Wang SJ, Ji P, Zhou GY, Zhao GP, Ge HL, Wang 
Y: dCTP pyrophosphohydrase exhibits nucleic accumulation in multiple carcinomas. European journal of histochemistry : EJH 2013, 57(3):e29.

84. Green ES: Analysis of HPV16 Variants in the Carolina Women's Care Study and a Comparison of Gene Expression Profiles of Exfoliated Cervical Cells From Women Who Either Clear or Do Not Clear an HPV16 Infection. (Doctoral dissertation). 2019.

85. Meng L, Dian F, Yinan X, Hao Z, Dingyue Z: Anticancer Effect of Natural Product Sulforaphane by Targeting MAPK Signal through miRNA-1247-3p in Human Cervical Cancer Cells. Biointerface Research in Applied Chemistry 2020, 11(1):7943-7972.

86. Wang X, Wang Y, Zhang Z, Huang M, Fei Y, Ma J, Mi L: Discriminating different grades of cervical intraepithelial neoplasia based on label-free phasor fluorescence lifetime imaging microscopy. Biomedical optics express 2020, 11(4):1977-1990.

87. Das P, Bansal A, Rao SN, Deodhar K, Mahantshetty U, Shrivastava SK, Sivaraman K, Mulherkar R: Somatic Variations in Cervical Cancers in Indian Patients. PLoS One 2016, 11(11):e0165878.

88. Li P, Guo H, Zhou G, Shi H, Li Z, Guan X, Deng Z, Li S, Zhou S, Wang Y et al: Increased ZNF84 expression in cervical cancer. Archives of gynecology and obstetrics 2018, 297(6):1525-1532.

89. Network CGAR: Integrated genomic and molecular characterization of cervical cancer. Nature 2017, 543(7645):378-384.

90. Wilson CM, Magnaudeix A, Yardin C, Terro F: DC2 and keratinocyte-associated protein 2 (KCP2), subunits of the oligosaccharyltransferase complex, are regulators of the gamma-secretase-directed processing of amyloid precursor protein (APP). The Journal of biological chemistry 2011, 286(36):31080-31091.

91. Huang HS, Buck CB, Lambert PF: Inhibition of gamma secretase blocks HPV infection. Virology 2010, 407(2):391-396.

92. Ivansson EL, Juko-Pecirep I, Erlich HA, Gyllensten UB: Pathway-based analysis of genetic susceptibility to cervical cancer in situ: HLA-DPB1 affects risk in Swedish women. Genes and immunity 2011, 12(8):605-614.

93. Manor O, Segal E: Robust Prediction of Expression Differences among Human Individuals Using Only Genotype Information. Plos Genet 2013, 9(3):e1003396.

94. Manor O, Segal E: GenoExp: a web tool for predicting gene expression levels from single nucleotide polymorphisms. Bioinformatics 2015, 31(11):1848-1850.

95. Yuan Z, Zhu H, Zeng P, Yang S, Sun S, Yang C, Liu J, Zhou X: Testing and controlling for horizontal pleiotropy with probabilistic Mendelian randomization in transcriptome-wide association studies. Nat Commun 2020, 11(1):3861. 
799

800

801

802

803
96. Yeung K-F, Yang Y, Yang C, Liu J: CoMM: A Collaborative Mixed Model That Integrates GWAS and eQTL Data Sets to Investigate the Genetic Architecture of Complex Traits. Bioinformatics and Biology Insights 2019, 13:1177932219881435. 
805 Figure 1. Schematic framework of our proposed two-stage causal inference approach.

806 Top: estimate the weight of each methylation site based on the methylation-expression

807 pair of a given gene with various prediction models; Bottom: evaluate the association

808 between methylation-regulated gene expression (MReX) and the survival of cervical

809 cancer using the Cox linear mixed-effects model and then discover causal genes for 810 cervical cancer in TCGA.

811 Figure 2. Flowchart for the present study with datasets of cervical cancer available 812 from TCGA. (1) Various levels of raw datasets were included for cervical cancer; we 813 conducted a series of quality control for those raw datasets; (2) gene expressions 814 predicted with methylations were generated with diverse prediction models, the Cox 815 linear mixed-effects model was applied to identify methylation-driven genes based on 816 predicted expression levels; we aggregated the $p$ values of genes from different 817 prediction models through a $p$-values combination manner to find significant genes 818 that were related to the survival of cervical cancer. Finally, we further implemented 819 functional and differential expression analyses for newly identified associated genes.

820 Figure 3. (A) The number of prediction models that have maximum $R^{2}$ across all the 821 genes analyzed when predicting expression level with using methylations. (B) 822 Pearson's correlation of the $p$ values (in a scale of - $\log 10$ ) obtained via in the Cox 823 linear mixed-effects model with five different prediction models. In the plot the 824 intensity of the color and the size of the circle represent the magnitude of the 825 correlation. (C) UpSet plot to illustrate the intersection of associated genes identified 826 by tests with five prediction models. LMM: Linear mixed model; BSLMM: Bayesian 827 sparse linear mixed model; DPR: Latent Dirichlet Process Regression; ENET: elastic 828 net; HMP: harmonic mean p-value combination method.

829 Figure 4. Manhattan plot showing the significance of all genes. Each plot is in a $830-\log 10$ (false discovery rate [FDR]) scale. Genes with $-\log 10$ FDR > 1.3 (i.e. FDR < 831 0.05) are highlighted. DPR: Latent Dirichlet Process Regression; ENET: elastic net; 832 HMP: harmonic mean p-value method 
833 Figure 5. (A) Heatmap of expression levels for these 23 newly identified causal genes 834 of cervical cancer. (B) Heatmap for differentially expressed genes. (C) Gene ontology 835 (GO) and Kyoto Encyclopedia of Genes and Genomes (KEGG) pathway enrichment 836 analyses for the 23 genes. Count Number denotes the number of genes related to the 837 enriched GO or KEGG pathway. 


\section{Figures}

\section{stage 1. Weight estimation for methylations via prediction models}

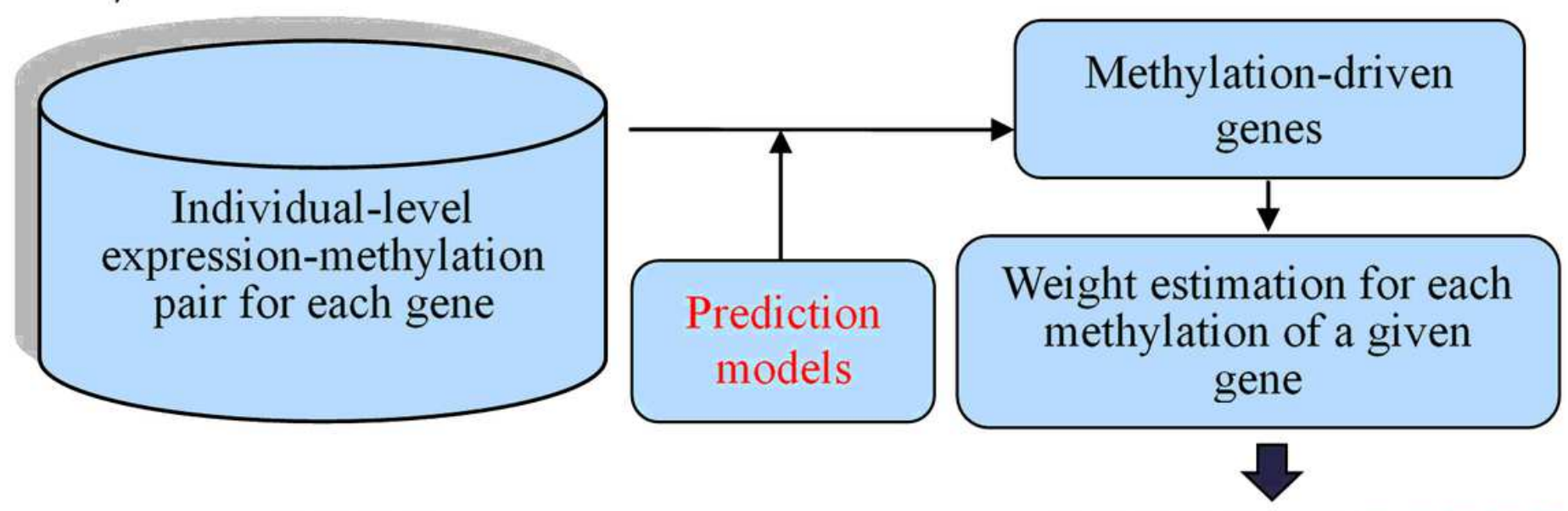

stage 2. Expression imputation and causal association identification

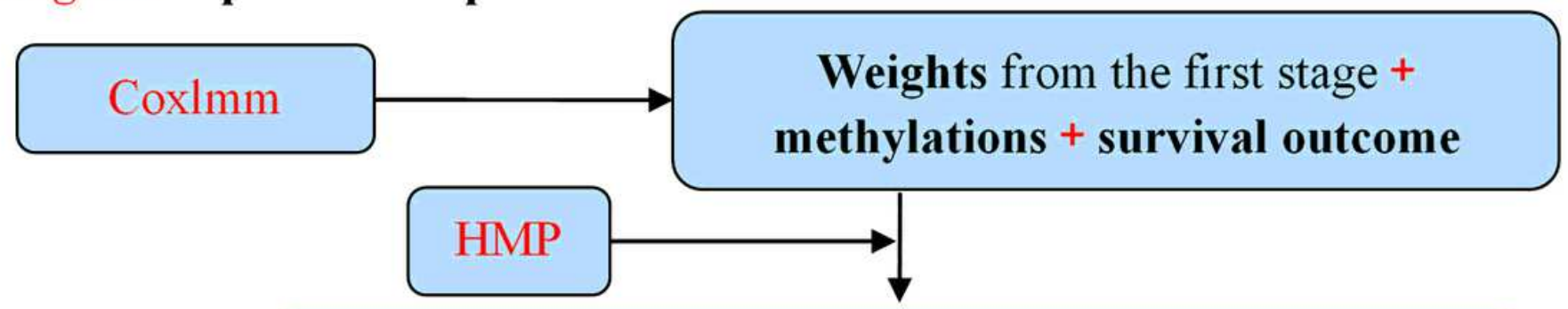

\section{Potential causal genes associated with the survival risk \\ of cervical cancer}

Figure 1

Schematic framework of our proposed two-stage causal inference approach. Top: estimate the weight of each methylation site based on the methylation-expression pair of a given gene with various prediction models; Bottom: evaluate the association between methylation-regulated gene expression (MReX) and the survival of cervical cancer using the Cox linear mixed-effects model and then discover causal genes for cervical cancer in TCGA. 


\section{Stage 1. TCGA data and quality control}

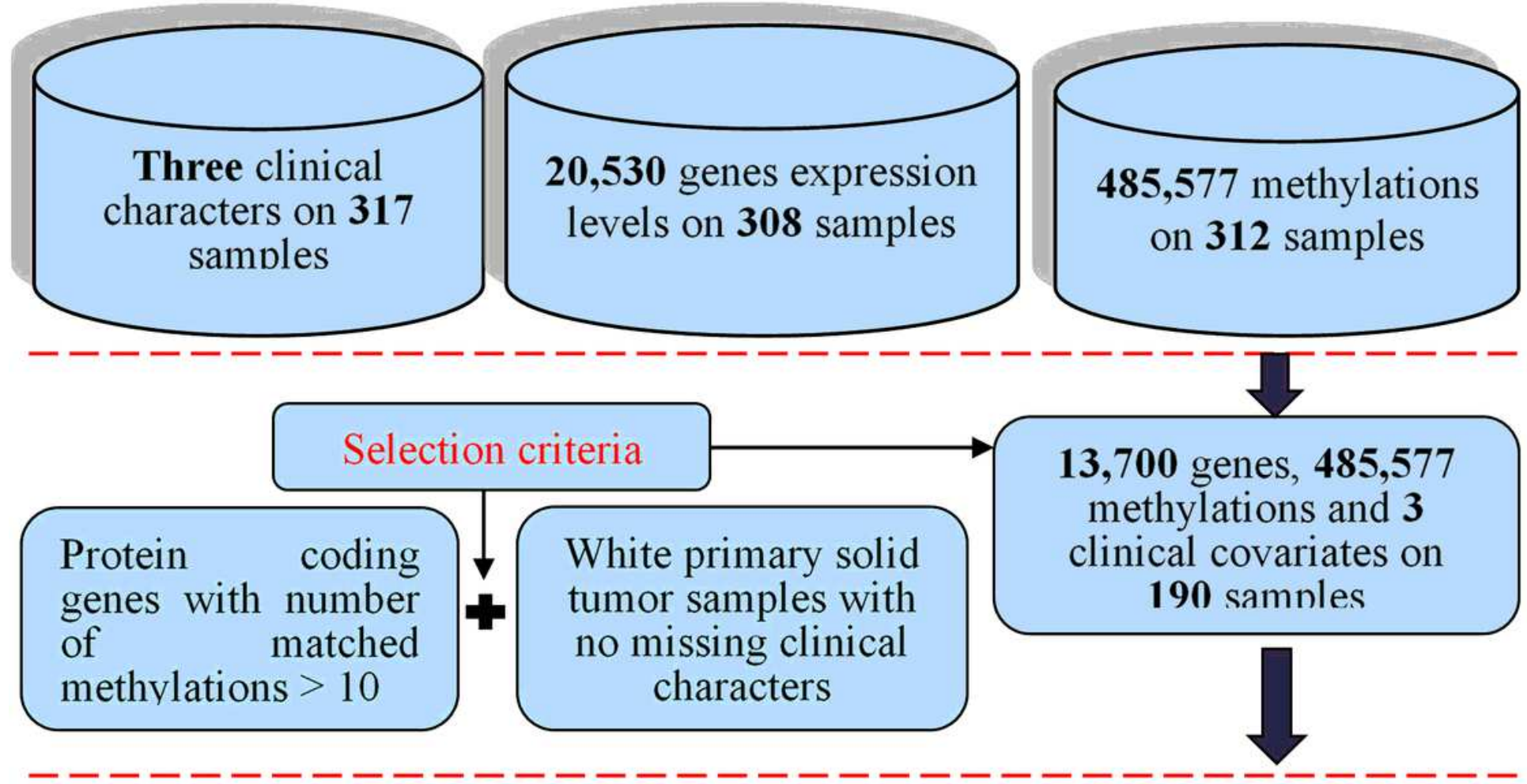

\section{Stage 2. Statistical analysis}

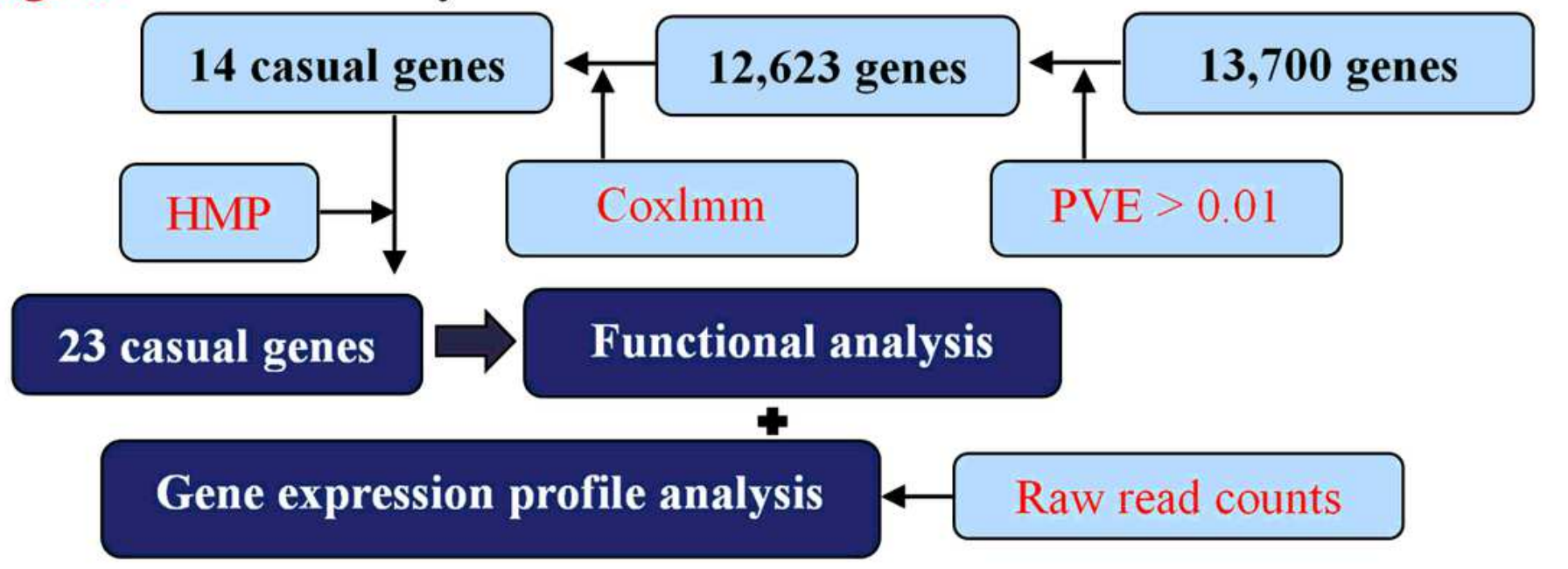

Figure 2

Flowchart for the present study with datasets of cervical cancer available from TCGA. (1) Various levels of raw datasets were included for cervical cancer; we conducted a series of quality control for those raw datasets; (2) gene expressions predicted with methylations were generated with diverse prediction models, the Cox linear mixed-effects model was applied to identify methylation-driven genes based on predicted expression levels; we aggregated the $p$ values of genes from different prediction models 
through a p-values combination manner to find significant genes that were related to the survival of cervical cancer. Finally, we further implemented functional and differential expression analyses for newly identified associated genes.

A

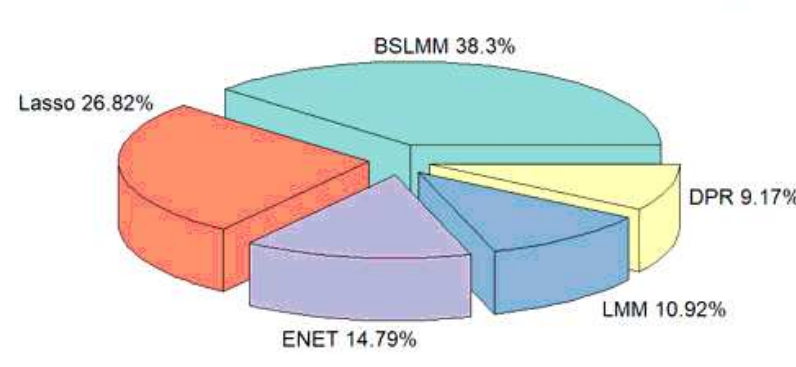

B

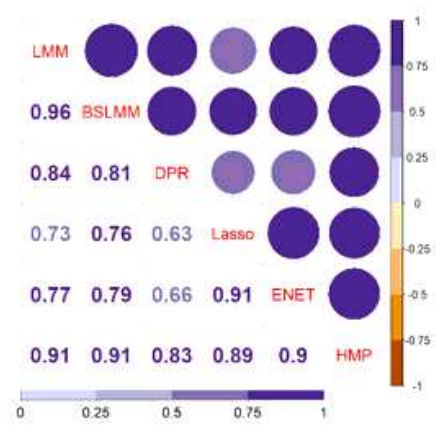

C

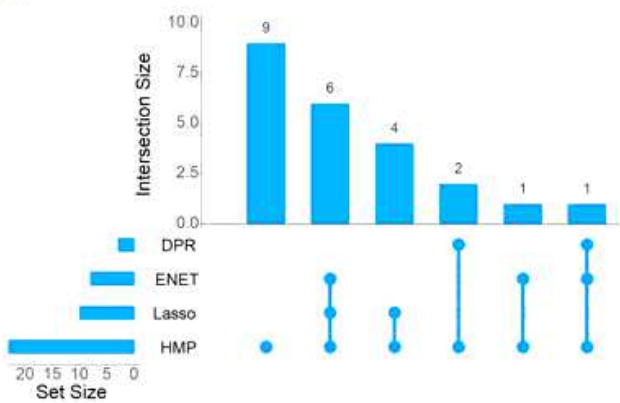

Figure 3

(A) The number of prediction models that have maximum R2 across all the genes analyzed when predicting expression level with using methylations. (B) Pearson's correlation of the $p$ values (in a scale of -log10) obtained via in the Cox linear mixed-effects model with five different prediction models. In the plot the intensity of the color and the size of the circle represent the magnitude of the correlation. (C) UpSet plot to illustrate the intersection of associated genes identified by tests with five prediction models. LMM: Linear mixed model; BSLMM: Bayesian sparse linear mixed model; DPR: Latent Dirichlet Process Regression; ENET: elastic net; HMP: harmonic mean p-value combination method.
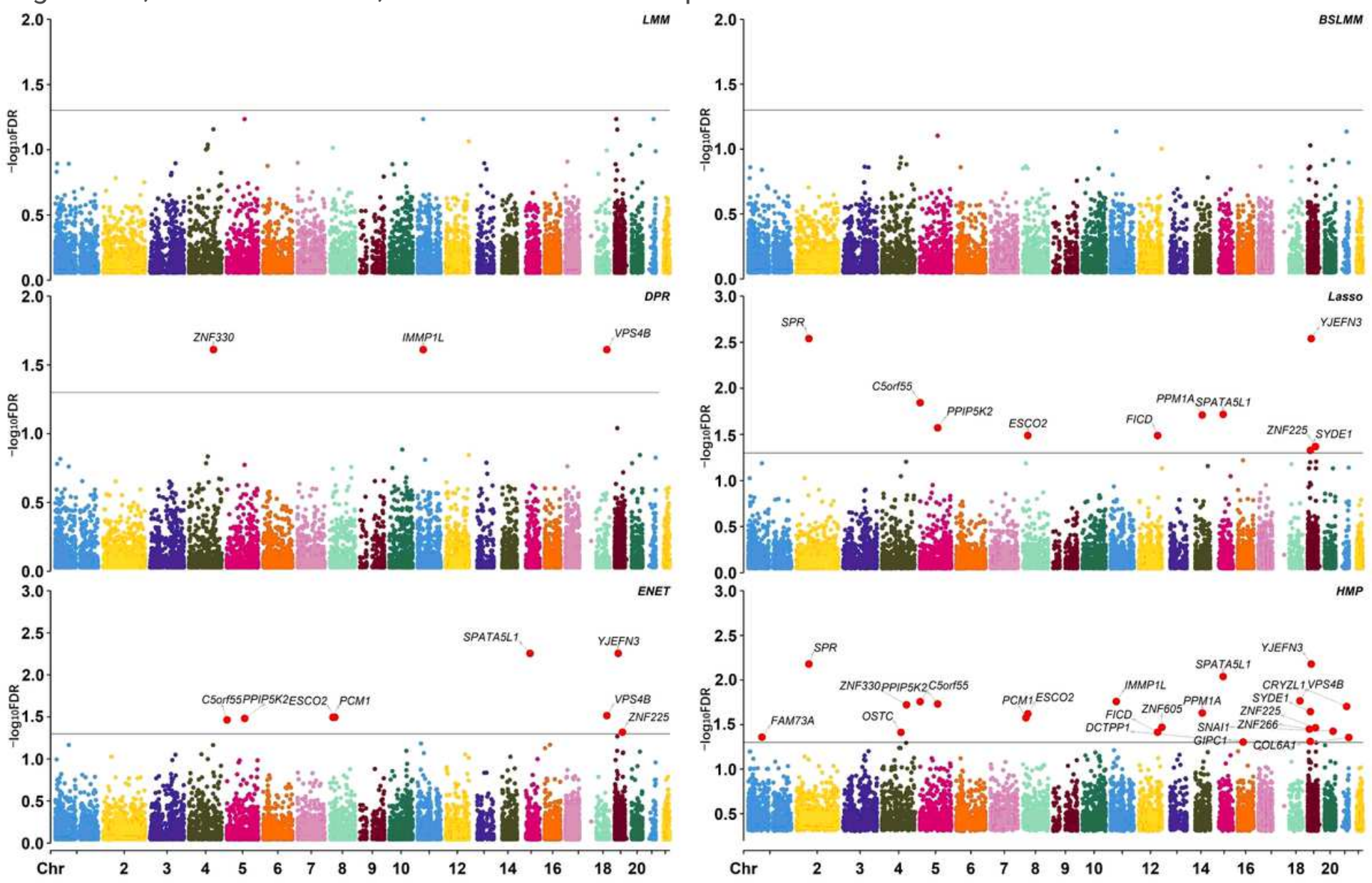
Figure 4

Manhattan plot showing the significance of all genes. Each plot is in a - $\log 10$ (false discovery rate [FDR]) scale. Genes with -log10 FDR > 1.3 (i.e. FDR < 0.05) are highlighted. DPR: Latent Dirichlet Process

Regression; ENET: elastic net; HMP: harmonic mean p-value method
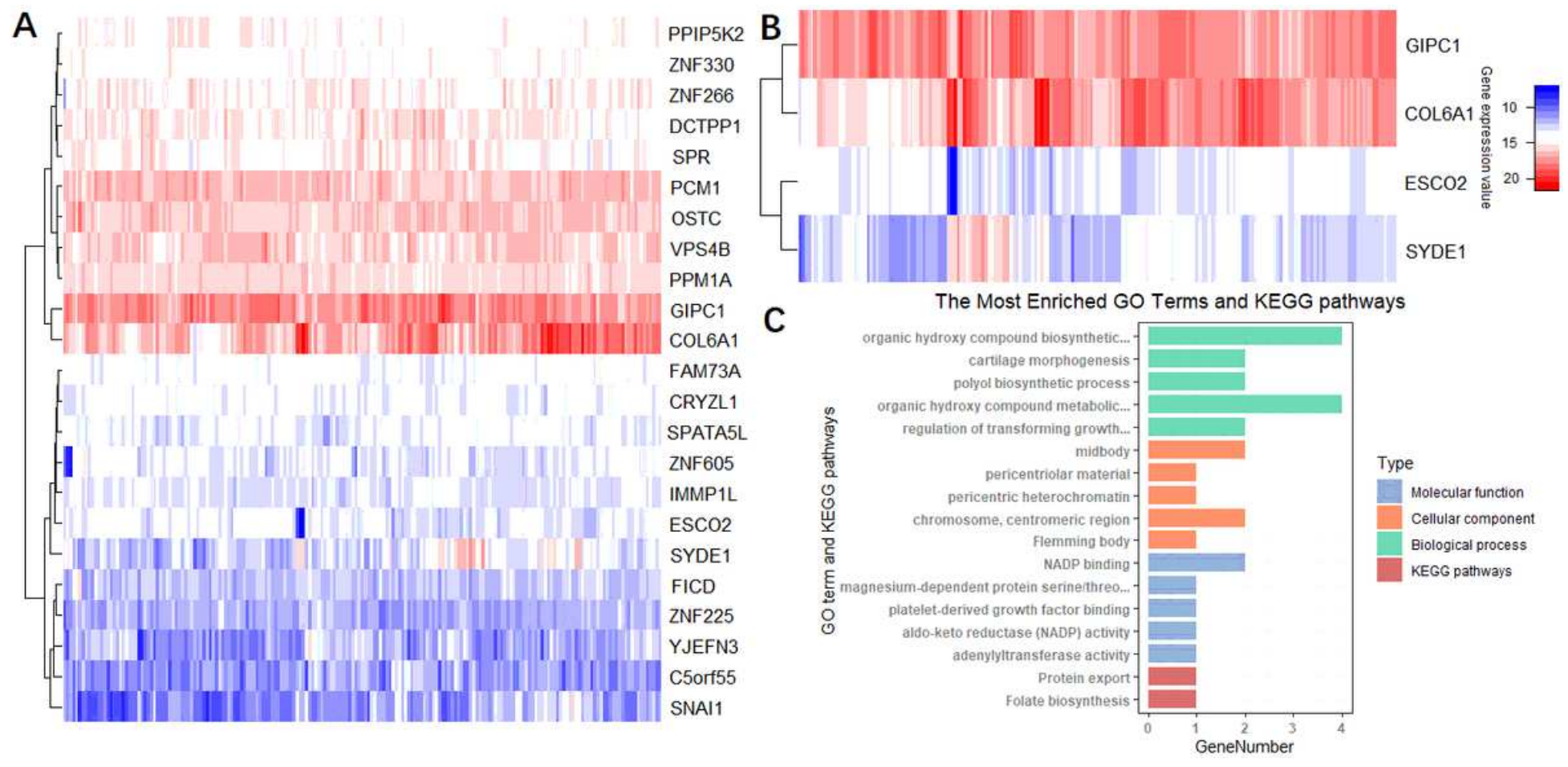

Figure 5

(A) Heatmap of expression levels for these 23 newly identified causal genes of cervical cancer. (B) Heatmap for differentially expressed genes. (C) Gene ontology (GO) and Kyoto Encyclopedia of Genes and Genomes (KEGG) pathway enrichment analyses for the 23 genes. Count Number denotes the number of genes related to the enriched GO or KEGG pathway.

\section{Supplementary Files}

This is a list of supplementary files associated with this preprint. Click to download.

- 20200911SupplementaryZhang.docx 